ASEAN Journal of Chemical Engineering 2021, Vol. 21, No. 1, 62 - 72

\title{
The Effect of Amino-functionalization on Photoluminescence Properties of Sugarcane Bagasse-derived Carbon Quantum \\ Dots
}

\author{
Muhammad Wahyu Nugraha ${ }^{1}$ \\ Nonni Soraya Sambudi ${ }^{1,2^{*}}$ \\ Laksmi Dewi Kasmiarno ${ }^{3}$ \\ Norashikin Ahmad Kamal 4 \\ ${ }^{1}$ Department of Chemical Engineering, Universiti Teknologi PETRONAS, Bandar Seri Iskandar, \\ Perak 32610, Malaysia \\ ${ }^{2}$ Centre of Urban Resource Sustainability (CUReS), Universiti Teknologi PETRONAS, Seri \\ Iskandar, Perak, 32610, Malaysia \\ ${ }^{3}$ Department of Chemical Engineering, Universitas Pertamina, Simprug, Jakarta Selatan, 12220, \\ Indonesia \\ ${ }^{4}$ Department of Civil Engineering, Universiti Teknologi MARA (UiTM) Shah Alam, Selangor, 40450, \\ Malaysia \\ `e-mail: soraya.sambudi@utp.edu.my
}

Submitted 12 November $2020 \quad$ Revised 20 March $2021 \quad$ Accepted 19 May 2021

\begin{abstract}
In the present study, amino-functionalized carbon quantum dots (N-CQDs) were prepared from sugarcane bagasse using a simple one-pot hydrothermal method. Both ethylenedinitrilotetraacetic (EDTA) \& ethylenediamine (EDA) were used as carbon and amino sources, respectively. The emerging utilization of natural carbon precursors is critically essential considering its low cost, eco-friendly, and unexploited by-products (e.g., sugarcane bagasse), which may have sustainable economic and strategic benefits. The as-prepared N-CQDs were characterized using High-Resolution Transmission Electron Microscope (HRTEM), Fourier Transform Infrared Spectroscopy (FTIR), UV-vis absorption spectroscopy, and photoluminescence spectroscopy. The influences of amine groups were investigated. The as-prepared N-CQDs photoluminescence intensity increased and quenched significantly with EDTA and EDA aminofunctionalization, respectively, was the highest quantum yield at $21.21 \%, 2.4$ times higher than nonfunctionalized CQDs. Furthermore, the amino-functional groups can alter the CQDs structure and particle size from $4.197 \pm 1.058 \mathrm{~nm}$ to $9.704 \pm 1.428 \mathrm{~nm}$. Which is, the N-CQDs produced exhibit highly tunable photoluminescence and particle size, which are potentially applicable in diverse applications.
\end{abstract}

Keywords: Amino-functionalization, Carbon quantum dots, Quantum yield, Sugarcane bagasse, Tunable

\section{INTRODUCTION}

Carbon quantum dots (CQDs) are spherical - carbon with a total size of less than $10 \mathrm{~nm}$ and have photophysical properties. Currently, a wide range of processes have been successful in producing CQDs. Nonetheless, most methods experienced unsatisfactory yield, extensive equipment setup, intricate procedures, and 
harsh conditions (Liao et al., 2016). There have been reports on the CQDs production using a simple one-pot hydrothermal process in which small crystal size, narrow size distribution, good crystallinity, and high photoluminescence CQDs were produced (Thambiraj, S., and Shankaran, D. R., 2016). Furthermore, the introduction of the passivation agent in CQDs e.g., aminofunctionalization $\left(\mathrm{NH}_{2}\right)$ enriched molecules, tuned the fluorescent performance and quantum yield (Wu et al., 2014). There are two roles of amino functionalization reported; it acts as a 1) $\mathrm{N}$-doping of precursor and 2) surface passivation agent (Zhai et al., 2012).

Synthesis of CQDs using natural carbon precursors is highly preferable, considering the cheap, environmentally friendly, and unutilized by-products. One of the main crops cultivated was sugarcane, with approximately $\sim 1.6$ billion tons of annual world production. This crop generates roughly $\sim 279$ million metric tons (MMT) of biomass residues (bagasse and leaves) (Chandel et al., 2012). Therefore, sugarcane bagasse (SB) has been explored in many applications. Eslami et al. (2018) successfully prepared CQDs from SB for naphthalene removal. They used an activation method using $\mathrm{KOH}$ activator followed by pyrolysis, which exhibited higher surface area with uniform pore size distribution (Eslami et al., 2018). Additionally, Thambiraj, S., and Shankaran, D. (2016) produced CQDs from sugarcane bagasse with a high quantum yield, approximately at $18.7 \%$.

Besides the enhancement of small-scale crystal size, confined size distribution, excellent crystallinity, and high photoluminescence level, the CQDs derived from natural precursors can be seen as an attractive characteristic, as observed in previous studies (Thambiraj, S., and
Shankaran, D., 2016). They have tunable photoluminescence that can be used for various applications such as sensors, bioimaging, drug delivery, sensing, and catalysis (Das, R., Bandyopadhyay, R., and Pramanik, P., 2018). The broad and tunable photoluminescence of CQDs mainly depends on the surface defect, and it can be tuned by using the passivation process on carbon precursors (Wu et al., 2014).

Thus, this current investigation studied the effects of amino-functionalization on the CQDs synthesized from sugarcane bagasse as a carbon precursor by using a simple one-pot hydrothermal method to see the effect on its crystal size, photoluminescence, size distribution, and quantum yield properties.

\section{MATERIALS AND METHOD}

\section{Materials}

Sugarcane bagasse was acquired from a nearby market in Seri Iskandar, Perak, Malaysia. Sodium hydroxide pellets (CAS 1310-73-2); ethylenediamine (CAS 107-15-3); Titriplex ${ }^{\circledR}$ III (ethylenedinitrilotetraacetic acid disodium salt dihydrate) (CAS 6381-92-6) were obtained from Merck (Germany). All chemicals were analytical reagent grade and used without any further purification or treatment. The deionized (DI) water (18.2 M $\Omega$ ) from PureLab Flex was employed for the entire experiment.

\section{Synthesis of Amino-Functionalized Carbon Quantum Dots}

The sugarcane CQDs were synthesized from the bagasse using a one-pot hydrothermal method, which has been published by Liu with some modifications (Liu et al., 2013). Firstly, the SB was sliced into dice pieces and then washed with deionized water. The wet-pieces of SB then sun-dried 
64 The Effect of Amino-functionalization on Photoluminescence Properties of Sugarcane Bagassederived Carbon Quantum Dots

until completely dried. Secondly, the dried SB was burned in a muffle furnace at $600^{\circ} \mathrm{C}$ for 1 hour to obtain the SB biochar.

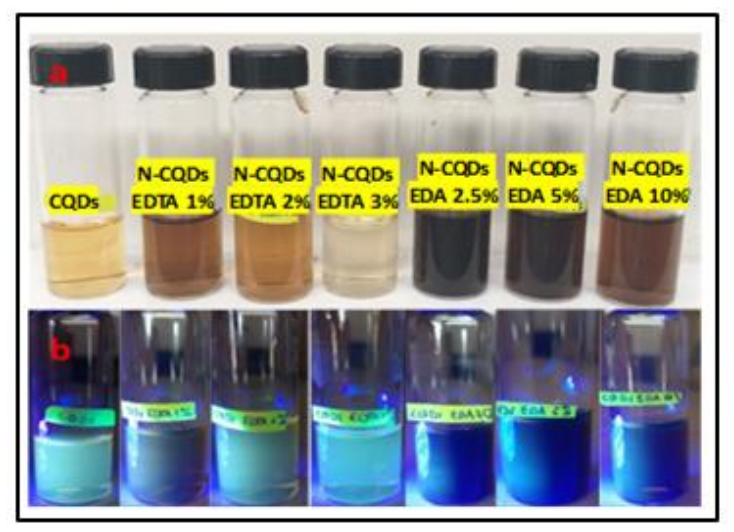

Figure 1. CQDs and N-CQDs solution appearance (a) under daylight irradiation (b) under UV-light irradiation

Subsequently, $300 \mathrm{mg}$ of SB biochar was added into $30 \mathrm{~mL}$ of $0.5 \mathrm{M}$ sodium hydroxide under constant stirring. The mixture was then heated in a Teflon-lined stainless-steel autoclave for hydrothermal processes at a temperature of $190^{\circ} \mathrm{C}$ for 24 hours. Afterward, the solution was cooled to room temperature, and the CQDs solution was vacuum filtrated using a $0.45 \mu \mathrm{m}$ PVDF membrane. The residue was dried separately at $60^{\circ} \mathrm{C}$. The CQDs solutions were then dialyzed using a dialysis membrane (MWCO3500) overnight.

In this study, the N-CQDs were synthesized in the same way as the CQDs synthesized method in the presence of varied concentrations of functionalization agent; EDA $(2.5-10 \% \mathrm{v} / \mathrm{v})$, and EDTA $(1-3 \% \mathrm{w} / \mathrm{w})$ in which it was mixed under vigorous stirring.

\section{Characterization}

The CQDs functional group was obtained using Fourier Transform Infrared Spectroscopy (FTIR) Perkin Elmer Spectrum One. Photoluminescence spectra and UV-
Visible spectra were documented using Maya2000 Pro Spectrometer with $390 \mathrm{~nm}$ excitation wavelength and Cary Series UV-Vis Spectrophotometer, respectively. Imaging of samples was performed using HighResolution Transmission Electron Microscope (HRTEM) $200 \mathrm{kV}$ with Field Emission, Tecnai G2 20 S-Twin, FEl, and the average particle sizes were determined using ImageJ software. Quantum yield (QY) of as-prepared CQDs was measured and compared quantitatively using the following Eq. (1) (Qu et al., 2016).

$Q Y=Q Y_{R} \times \frac{I_{S}}{I_{R}} \times \frac{A_{R}}{A_{S}} \times \frac{\eta^{2} s}{\eta^{2}{ }_{R}}$

Where $R$ represents as reference and $S$ represents as sample. The $I, A$, and $\eta$ are the photoluminescence intensity area, absorbance value, and refractive index of the solvent, respectively. The reference used was QY of quinine sulfate at $0.1 \mathrm{M} \mathrm{H}_{2} \mathrm{SO}_{4}$ ( $\eta=$ 1.33), which was 0.54 with an excitation wavelength of $350 \mathrm{~nm}$ (Chandra et al., 2013).

\section{RESULTS AND DISCUSSION}

\section{Morphology and functional groups of CQDs}

Different compositions of aminofunctionalization affected the appearance of $\mathrm{N}-\mathrm{CQDs}$ solution. The CQDs solution appeared bright yellow, while N-CQDs with EDTA had slightly darker bright yellow tones and gradually became lighter with the higher EDTA composition. On the other hand, the NCQDs EDA had a dark brown appearance and gradually became lighter brown in a higher EDA under daylight irradiation as shown in Figure 1a. Apart from that, the samples were examined under UV-light irradiation (Figure 1b). The CQDs emitted light green colour, while N-CQDs EDTA appeared as dark green and gradually shifted to a lighter blue colour 
with higher EDTA concentrations. The NCQDs EDA emitted a dark blue colour and consistently shifted to a lighter blue colour with higher EDA concentrations.

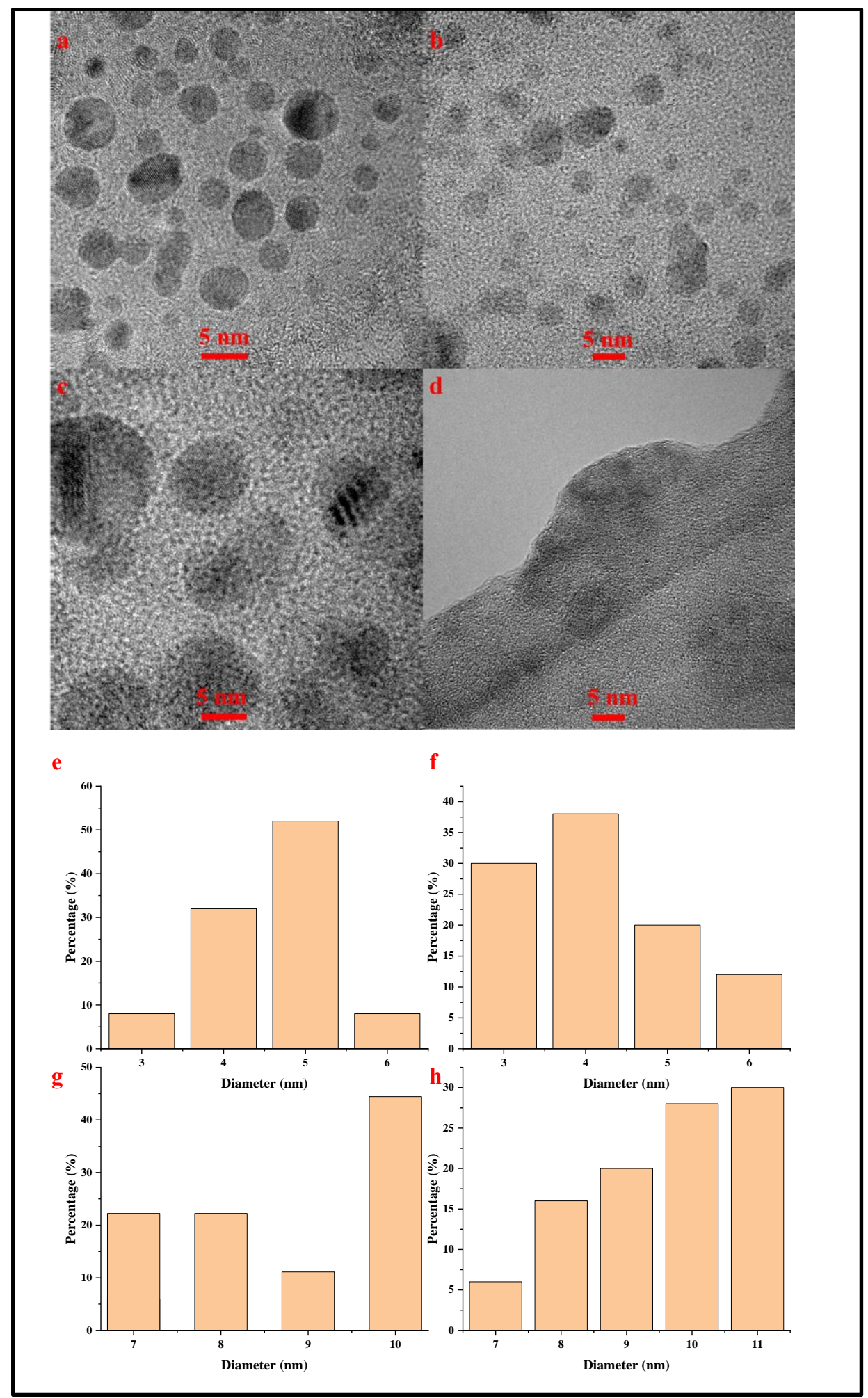

Figure 2. HRTEM imaging (a) N-CQDs EDTA 2\%, (b) N-CQDs EDTA 3\% (c) N-CQDs EDA 5\% (d) N-CQDs EDA 10\%, Size distribution (e) N-CQDs EDTA 2\%, (f) N-CQDs EDTA 3\% (g) N-CQDs EDA $5 \%$ (h) N-CQDs EDA $10 \%$ 
66 The Effect of Amino-functionalization on Photoluminescence Properties of Sugarcane Bagassederived Carbon Quantum Dots

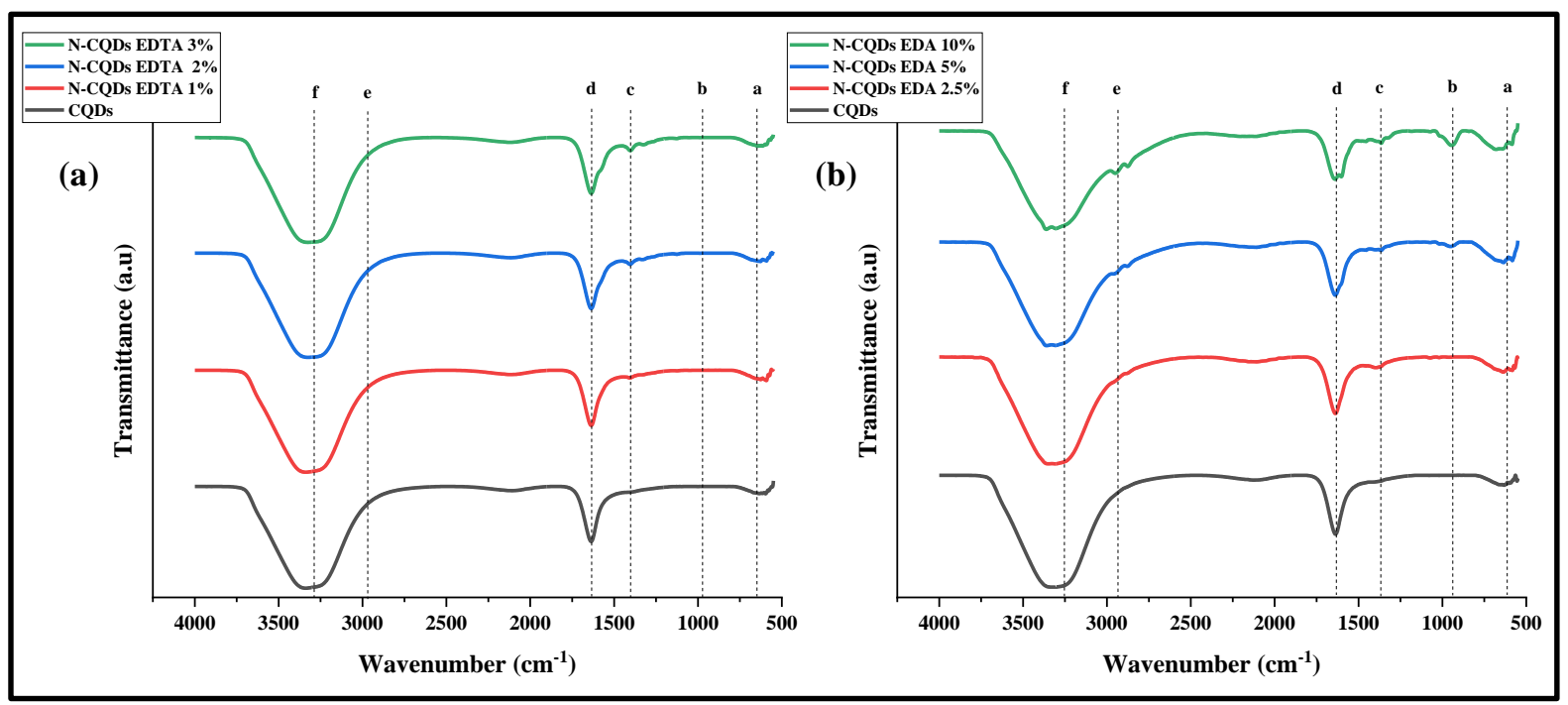

Figure 3. (a) FTIR spectra of N-CQDs EDTA (b) FTIR spectra of N-CQDs EDA

Moreover, the HRTEM imaging and size distribution (Figure 2) indicated spherical shape and excellent dispersion of CQDs with relatively uniform size distribution. The size of selected CQDs was calculated by measuring the diameter of 50 particles. The diameter size of N-CQDs EDTA $2 \%$ ranges from 3.88 to $5.33 \mathrm{~nm}$ with a lattice spacing of $0.207 \mathrm{~nm}$. While the N-CQDs EDTA 3\% diameter ranges from 3.13 to $5.25 \mathrm{~nm}$ with a lattice spacing of $0.209 \mathrm{~nm}$. The diameter of N-CQDs decreased with the higher EDTA functionalization. In contrast, both N-CQDs EDA $5 \%$ and $10 \%$ had a higher average diameter range than the EDTA functionalized CQDs, at approximately $8.27-11.13 \mathrm{~nm}$ with $0.202 \mathrm{~nm}$ lattice spacing and 6.87-10.91 nm with $0.310 \mathrm{~nm}$ lattice spacing, respectively. The higher EDA concentration resulted in a lower diameter. Thus, the aminofunctionalization could alter to lower size CQDs by edge-termination with higher amine concentrations which is similarly seen in the previous study (Tetsuka et al., 2012). The mentioned particle size demonstrated different fluorescence intensities (Figure 4) of CQDs. N-CQDs EDTA $3 \%$ had the smallest particle size which emitted the highest photoluminescence among the samples. In contrast, N-CQDs EDA $2.5 \%$ had the lowest photoluminescence intensity as it had relatively larger particles (Chandra et al., 2012).

The functional groups of N-CQDs samples were identified using FTIR spectra (Figure 3) to identify the amino functionalization on CQDs. The broad peaks at $3200-3500 \mathrm{~cm}^{-1}$ (peak $f$ ) are attributed to $-\mathrm{OH}$ bending vibrations on the surface of CQDs which indicated that the N-CQDs are rich for the oxygen-containing group. Thus, as-prepared CQDs have hydrophilic property and good stability $\&$ dispersibility in water (Liu, Y. et al., 2015). While peaks at 1637 and $992 \mathrm{~cm}^{-1}$ (peak $\mathrm{d}$ and $\mathrm{b}$ ) correspond to stretching vibration of $\mathrm{C}=\mathrm{O}$, and $\mathrm{C}-\mathrm{H}$, respectively. Furthermore, the effect of the aminofunctionalization agent is observed in the peak spectra. The amino-containing groups are observable at 1470 , and $2800 \mathrm{~cm}^{-1}$ (peak 
c and e) which correspond to the $\mathrm{C}-\mathrm{NH}$ stretching and $\mathrm{N}-\mathrm{H}$ bending vibration, respectively (Liu, X., et al., 2016; Madrakian et al., 2017). When the amount of the functionalization agent increases, the absorption peaks observed increased.

However, the N-CQDs EDTA peak of 2800 $\mathrm{cm}^{-1}$ (peak e) which corresponds to aminofunctionalization of CQDs is not detected. That is due to the lower concentrations of amino-functionalized in N-CQDs EDTA compared to N-CQDs EDA. Furthermore, a similar trend was also observed by Ji et al., (2019) where CQDs were also prepared using EDTA. They observed that the presence of the $\mathrm{N}$-doped of N-CQDs EDTA only appeared at peaks around $1470-1550 \mathrm{~cm}^{-1}$ (Ji et al., 2019).

\section{UV-Vis Analysis}

The UV-vis absorption spectrum of CQDs and $\mathrm{N}-\mathrm{CQDs}$ aqueous solution in Figure $4 \mathrm{a}$ revealed the centered emission wavelength, which is observed at $236 \mathrm{~nm}$ attributed to the $\pi-\pi^{*}$ transition of aromatic $\mathrm{sp}^{2}$ domains (Freire et al., 2018). Furthermore, at approximately 350-700 $\mathrm{nm}$, another symmetrical and robust absorption band was observed with red-shift due to aminofunctionalization. The peak was also observed by Freire et al. (2018). This notable band is attributed to the $\mathrm{n}-\pi^{*}$ transition ( $\mathrm{C}-\mathrm{O}$ and $\mathrm{N}$ $\mathrm{H}$ groups), as well as contributions of the surface groups that resulted from amino edge functionalization (Freire et al., 2018; Sandeep Kumar et al., 2014). It can be seen that the amino-functionalization of EDTA and EDA broadened the absorption peak at the visible light range (400-700 nm). From Figure 4 , it can be seen that the aminofunctionalization red-shifted at initial concentration. However, at higher amino concentrations, the UV-vis spectra are blue- shifted but still maintain to have stronger visible light region absorption peak compared to pristine CQDs, except for $\mathrm{N}$ CQDs EDTA 3\%. The blue-shift emission is affects by the quantum confinement effect (QCE) of electrons inside the $-\mathrm{sp}^{2}$ carbon domains. Generally, the QCE effect is mainly affiliated with the size of nanoparticles. The small size affected the luminescence energy to be blue-shifted (Zainal Abidin et al., 2020). The as-prepared N-CQDs EDTA $3 \%$ is the smallest particle size compared to other asprepared N-CQDs. Thus, the blue-shift is more prominent. This trend was also observed in the previous study (Zainal Abidin et al., 2020).

\section{Photoluminescence Analysis}

The fluorescence emission spectra of amino-functionalized CQDs are observed for CQDs, N-CQDs EDTA, and N-CQDs EDA at $390 \mathrm{~nm}$ excitation. It is indicated that the EDTA has shown higher results for inducing highly fluorescent CQDs compared to EDA, which is quenched for the fluorescence intensity. Moreover, the fluorescence changes of the prepared N-CQDs were also observed. The results have shown that the increased concentration of EDTA from $1 \%$ to $3 \%$ enhanced the fluorescence intensity of $\mathrm{N}$ CQDs significantly. Conversely, the EDA immensely quenched N-CQDs' fluorescence intensity; however, among the samples with EDA loading, as the concentration increased from $2.5 \%$ to $10 \%$, the fluorescence intensity also increased, as shown in Figure $4 \mathrm{~b}$. The stability of carbon quantum dots is dependent on their photoluminescence property, which is consistent with surrounding molecules. It isolates the particle and prevents the non-radiative recombination through surface modification 
68 The Effect of Amino-functionalization on Photoluminescence Properties of Sugarcane Bagassederived Carbon Quantum Dots

(Chandra et al., 2012). Furthermore, when a particle is transmuted into a more isolated form, it contributed more to the photoluminescence intensity compared to its corresponding non-isolable particles (Chandra et al., 2012). This is because the particles could transfer energy between themselves when they are in close distance, thus diminishing their fluorescence properties (Chandra et al., 2012; Jameson, 2014).

Compared to Zhai et al. (2012) works which used various amine passivation agents including 1,2-ethylenediamine (EDA), diethylamine (DEA), triethylamine (TEA), and 1,4-butanediamine (BDA) to study the role of amino groups in the fabrication, the amino molecules are found to play two functions: $\mathrm{N}$ doping precursors and surface passivation agents for the CQDs. Both functions play a role in enhancing photoluminescence performance. Significantly, the as-prepared $\mathrm{N}-\mathrm{CQDs}$ have a higher quantum yield.

Furthermore, the maximum emission wavelength of the samples was observed using normalized intensity. After excitation at $390 \mathrm{~nm}$ wavelength, CQDs and N-CQDs EDTA $1 \%, 2 \%$, and $3 \%$ emitted maximum wavelength at 572, 525, 542, and $521 \mathrm{~nm}$, respectively. As-prepared CQDs emitted green photoluminescence after excitation. It is shown that the maximum emission wave-

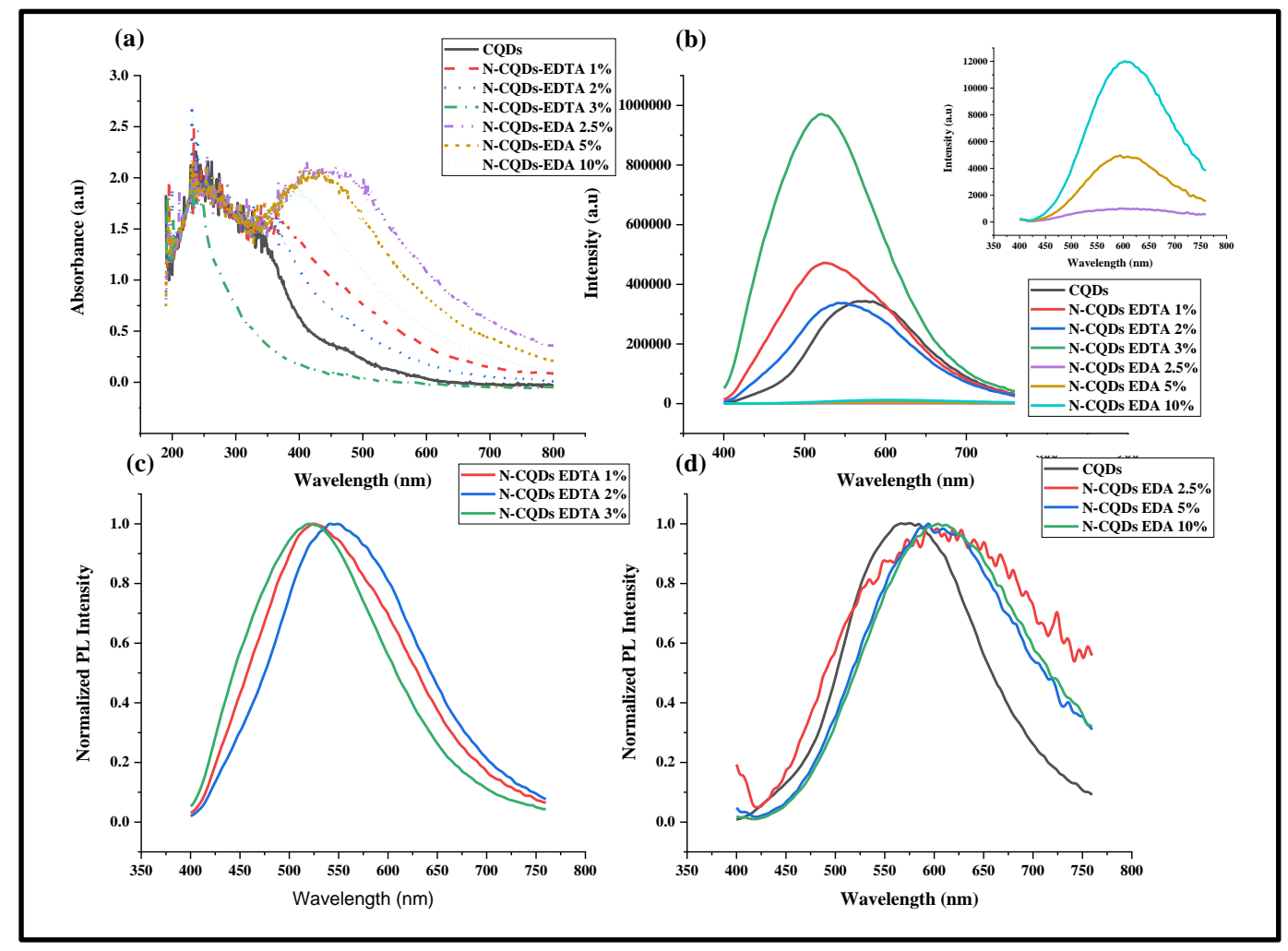

Fig. 4: (a) UV-Vis spectra, (b) Photoluminescence spectra with inset Photoluminescence spectra at lower intensity, (c), (d) Normalized PL spectra of N-CQDs samples 
Table 1. Comparison of CQDs from natural precursor

\begin{tabular}{|c|c|c|c|c|c|}
\hline Precursor & $\begin{array}{c}\text { Functionali } \\
\text { zation } \\
\text { Agent }\end{array}$ & $\begin{array}{c}\text { Particle Size } \\
\text { (nm) }\end{array}$ & $\begin{array}{l}\text { PL Intesity } \\
\text { (a.u) }\end{array}$ & Remarks & Ref. \\
\hline $\begin{array}{l}\text { Rice } \\
\text { residue }\end{array}$ & Lysine & 2.70 & $\approx 5500$ & $\begin{array}{l}\text { The application capability } \\
\text { of } \mathrm{N} \text {-CQDs has been } \\
\text { demonstrated for } \\
\text { detecting } \mathrm{Fe}^{3+} \text { ions and } \\
\text { tetracyclines. }\end{array}$ & $\begin{array}{l}\text { (Qi et al., } \\
\text { 2019) }\end{array}$ \\
\hline $\begin{array}{c}\text { Sugarcane } \\
\text { bagasse }\end{array}$ & - & $4.1 \pm 0.17$ & $\approx 550$ & QY $18.7 \%$ & $\begin{array}{c}\text { (S. \& D., } \\
2016)\end{array}$ \\
\hline $\begin{array}{l}\text { Sugarcane } \\
\text { molasses }\end{array}$ & - & $1.2-3.8$ & - & QY 5.8\% & $\begin{array}{l}\text { (Huang et } \\
\text { al., 2017) }\end{array}$ \\
\hline $\begin{array}{l}\text { Sugarcane } \\
\text { bagasse }\end{array}$ & $\begin{array}{c}\text { EDTA } \\
\text { EDA }\end{array}$ & $\begin{array}{l}4.197 \pm 1.058 \\
9.704 \pm 1.428\end{array}$ & $\begin{array}{l}970493.375 \\
1008.48669\end{array}$ & $\begin{array}{l}\text { The PL intensity of as- } \\
\text { prepared N-CQDs can be } \\
\text { tuned either to have } \\
\text { higher photoluminescence } \\
\text { or quenched } \\
\text { photoluminescence. The } \\
\text { highest QY recorded was } \\
21.21 \%\end{array}$ & This study \\
\hline
\end{tabular}

length blue-shifted to a shorter wavelength with a higher concentration of the EDTA passivation agent. Compared to pristine CQDs, the N-CQDs EDTA 3\% maximum emission peak sharply decreased from the green region photoluminescence to the blue region photoluminescence. On the other hand, EDA functionalization was observed differently. After excitation at $390 \mathrm{~nm}$, CQDs and N-CQDs EDA $2.5 \%, 5 \%$, and 10\% emitted maximum wavelength at $572,594,594$, and $605 \mathrm{~nm}$, respectively. It was red-shifted to a longer wavelength region photoluminescence at a higher EDA concentration. The application of broad photoluminescence of CQDs is favorable. It is potentially applicable for multicolor imaging that requires low toxicity in photocatalysts and solar cells as it extends the light absorption that might lead to higher performance. The photoluminescence CQDs were originated due to their distinct size (Jang et al., 2015;
Sarkar et al., 2016) and surface defect (Di et al., 2017; Ding et al., 2016). That is caused by functional group, edge effect, and disordered structure of CQDs.

The photoluminescence excitation spectrum of N-CQDs EDTA 3\% (521 nm) shows a blue-shift, and N-CQDs EDA 10\% (605 nm) is red-shifted compared to the pristine CQDs (572 nm). It can be concluded that the optical performance of N-CQDs is influenced by the amino-containing groups (Wu et al., 2014; Zhai et al., 2012). It can be tuned from greenish to the blue region using an EDTA functionalization agent and greenish to yellowish region using EDA, through an increasing amount of aminofunctionalization.

Additionally, the comparison of the produced N-CQDs with other studies that use natural precursors as carbon sources is presented in Table 1. The quantum yield (QY) of as-prepared CQDs are $8.88 \%, 11.77 \%$, 
70 The Effect of Amino-functionalization on Photoluminescence Properties of Sugarcane Bagassederived Carbon Quantum Dots

9.17\%, $21.21 \%, 0.02 \%, 0.10 \%$, and $0.27 \%$ for CQDs, N-CQDs EDTA 1\%, N-CQDs EDTA 2\%, $\mathrm{N}$-CQDs EDTA $3 \%, \mathrm{~N}-\mathrm{CQDS}$ EDA $2.5 \%, \mathrm{~N}$ CQDs EDA 5\%, and N-CQDs EDA 10\%, respectively. The as-prepared $\mathrm{N}-\mathrm{CQDs}$ have highly tunable photoluminescence and particle size.

\section{CONCLUSION}

$\mathrm{N}$-CQDs have been synthesized via a simple one-pot hydrothermal process. In this study, it has been reported that aminofunctionalization alters the functional groups on the surface, photoluminescence, and particle size of the as-prepared CQDs. Furthermore, the photoluminescence of $\mathrm{N}$ CQDs is immensely enhanced using a higher concentration of EDTA passivation agent and contrarily quenched using the EDA passivation agent, with the quantum yield reached $21.21 \%$, which is around 2.4 times higher than original CQDs. Lastly, the obtained N-CQDs shifted the emission wavelength of CQDs from greenish to blue photoluminescence using EDTA passivation agent. In contrast, it shifted from greenish to yellowish photoluminescence using EDA. The effect on photoluminescence and particle size in this study was originated from nitrogen content (amino functionalization agent amount) in CQDs. This study offers a simple way to produce tunable photoluminescence N-CQDs from sugarcane bagasse.

\section{ACKNOWLEDGEMENT}

This work is supported by Fundamental Research Grant Scheme (FRGS) project with code FRGS/1/2018/TK02/UTP/03/3.

\section{REFERENCES}

1. Chandel, A. K., da Silva, S. S., Carvalho, W., \& Singh, O. V. (2012). Sugarcane bagasse and leaves: Foreseeable biomass of biofuel and bio-products. Journal of Chemical Technology and Biotechnology, 87, 11-20.

2. Chandra, S., Pathan, S. H., Mitra, S., Modha, B. H., Goswami, A., \& Pramanik, P. (2012). Tuning of photoluminescence on different surface functionalized carbon quantum dots. RSC Advances, 2, 3602.

3. Chandra, S., Patra, P., Pathan, S. H., Roy, S., Mitra, S., Layek, A., ... Goswami, A. (2013). Luminescent S-doped carbon dots: an emergent architecture for multimodal applications. Journal of Materials Chemistry B, 1, 2375.

4. Das, R., Bandyopadhyay, R., \& Pramanik, P. (2018). Carbon quantum dots from natural resource: A review. Materials Today Chemistry, 8, 96-109.

5. Di, J., Xia, J., Chen, X., Ji, M., Yin, S., Zhang, Q., \& Li, H. (2017). Tunable oxygen activation induced by oxygen defects in nitrogen doped carbon quantum dots for sustainable boosting photocatalysis. Carbon, 114, 601-607.

6. Ding, H., Yu, S.-B., Wei, J.-S., \& Xiong, H.M. (2016). Full-Color Light-Emitting Carbon Dots with a Surface-StateControlled Luminescence Mechanism. ACS Nano, 10, 484-491.

7. Eslami, A., Borghei, S. M., Rashidi, A., \& Takdastan, A. (2018). Preparation of activated carbon dots from sugarcane bagasse for naphthalene removal from aqueous solutions. Separation Science and Technology, 53, 2536-2549.

8. Freire, R. M., Le, N. D. B., Jiang, Z., Kim, C. S., Rotello, V. M., \& Fechine, P. B. A. 
(2018). NH2-rich Carbon Quantum Dots:

A protein-responsive probe for detection and identification. Sensors and Actuators B: Chemical, 255, 2725-2732.

9. Huang, G., Chen, X., Wang, C., Zheng, H., Huang, Z., Chen, D., \& Xie, H. (2017). Photoluminescent carbon dots derived from sugarcane molasses: synthesis, properties, and applications. RSC Adv., 7, 47840-47847.

10. Jameson, D. M. (2014). Introduction to Fluorescence. In Introduction to Fluorescence. CRC Press.

11. Jang, M.-H., Ha, H. D., Lee, E.-S., Liu, F., Kim, Y.-H., Seo, T. S., \& Cho, Y.-H. (2015). Is the Chain of Oxidation and Reduction Process Reversible in Luminescent Graphene Quantum Dots? Small, 11, 3773-3781.

12. Ji, T., Fan, P., Li, X., Mei, Z., Mao, Y., \& Tian, Y. (2019). EDTA-bonded multi-connected carbon-dots and their Eu3+ complex: Preparation and optical properties. RSC Advances, 9, 10645-10650.

13. Liao, B., Wang, W., Deng, X., He, B., Zeng, W., Tang, Z., \& Liu, Q. (2016). A facile onestep synthesis of fluorescent silicon quantum dots and their application for detecting $\mathrm{Cu} 2+$. RSC Advances, 6, 14465-14467.

14. Liu, X. J., Guo, M. L., Huang, J., \& Yin, X. Y. (2013). Improved fluorescence of carbon dots prepared from bagasse under alkaline hydrothermal conditions. BioResources, 8, 2537-2546.

15. Liu, X., Pang, J., Xu, F., \& Zhang, X. (2016). Simple Approach to Synthesize AminoFunctionalized Carbon Dots by Carbonization of Chitosan. Scientific Reports, 6, 1-8.

16. Liu, Y., Liu, Y., Park, S.-J., Zhang, Y., Kim, T., Chae, S., ... Kim, H.-Y. (2015). One-step synthesis of robust nitrogen-doped carbon dots: acid-evoked fluorescence enhancement and their application in $\mathrm{Fe}$ $3+$ detection. Journal of Materials Chemistry A, 3, 17747-17754.

17. Madrakian, T., Maleki, S., Gilak, S., \& Afkhami, A. (2017). Turn-off fluorescence of amino-functionalized carbon quantum dots as effective fluorescent probes for determination of isotretinoin. Sensors and Actuators, B: Chemical, 247, 428-435.

18. Qi, H., Teng, M., Liu, M., Liu, S., Li, J., Yu, H., ... Guo, Z. (2019). Biomass-derived nitrogen-doped carbon quantum dots: highly selective fluorescent probe for detecting $\mathrm{Fe} 3+$ ions and tetracyclines. Journal of Colloid and Interface Science, 539, 332-341.

19. Qu, A., Xie, H., Xu, X., Zhang, Y., Wen, S., \& Cui, Y. (2016). High quantum yield graphene quantum dots decorated $\mathrm{TiO} 2$ nanotubes for enhancing photocatalytic activity. Applied Surface Science, 375, 230-241.

20. Sandeep Kumar, G., Roy, R., Sen, D., Ghorai, U. K., Thapa, R., Mazumder, N., ... Chattopadhyay, K. K. (2014). Aminofunctionalized graphene quantum dots: origin of tunable heterogeneous photoluminescence. Nanoscale, 6, 3384.

21. Sarkar, S., Banerjee, D., Ghorai, U. K., Das, N. S., \& Chattopadhyay, K. K. (2016). Size dependent photoluminescence property of hydrothermally synthesized crystalline carbon quantum dots. Journal of Luminescence, 178, 314-323.

22. Tetsuka, H., Asahi, R., Nagoya, A., Okamoto, K., Tajima, I., Ohta, R., \& Okamoto, A. (2012). Optically tunable amino-functionalized graphene quantum dots. Advanced Materials, 24, 5333-5338.

23. Thambiraj, S., and Shankaran, D. (2016). 
72 The Effect of Amino-functionalization on Photoluminescence Properties of Sugarcane Bagassederived Carbon Quantum Dots

Green synthesis of highly fluorescent carbon quantum dots from sugarcane bagasse pulp. Applied Surface Science, 390, 435-443.

24. Wu, M., Wang, Y., Wu, W., Hu, C., Wang, X., Zheng, J., ... Qiu, J. (2014). Preparation of functionalized water-soluble photoluminescent carbon quantum dots from petroleum coke. Carbon, 78, 480489.

25. Zainal Abidin, N. H., Wongso, V., Hui, K. C., Cho, K., Sambudi, N. S., Ang, W. L., \& Saad, B. (2020). The effect of functionalization on rice-husks derived carbon quantum dots properties and cadmium removal. Journal of Water Process Engineering, 38, 101634.

26. Zhai, X., Zhang, P., Liu, C., Bai, T., Li, W., Dai, L., \& Liu, W. (2012). Highly luminescent carbon nanodots by microwave-assisted pyrolysis. Chemical Communications, 48, 7955. 